\title{
Estudios
}

\section{Eucaristía y praxis cristiana: Reflexiones en diálogo con Edith Stein}

\author{
Fernando Berrios \\ Facultad de Teología \\ Pontificia Universidad Católica de Chile \\ fberriosm@uc.cl
}

Resumen: En Das Gebet der Kirche -La oración de la Iglesia-, texto escrito en 1936 en el Carmelo de Colonia, Alemania, Edith Stein expresa su vivencia y su comprensión de la oración, de la liturgia y del culmen de ambas en la Eucaristía. Se ha destacado este escrito como un verdadero testamento espiritual en el que la autora profundiza y vincula, desde sus raíces en la tradición judía, el sentido de la oración como adhesión íntima y personal a Jesucristo, de la eucaristía como experiencia fundante de esa comunión en la Iglesia, y de una praxis cristiana en clave sacrificial como momento interno y verificación necesaria de esa vivencia. El presente trabajo destacará tres contenidos básicos del texto: (1.) la oración del cristiano es siempre un acto "aprendido" de la oración de Cristo, que es su "modelo" por antonomasia; (2.) la oración es siempre oración de la Iglesia y no simplemente un acto individual; y por último, (3.) ella tiende siempre a una acción, como signo indefectible y necesario de lo que Dios obra en la vida espiritual de sus fieles.

Palabras clave: Edith Stein - Oración - Iglesia - Eucaristía - Acción.

Abstract: In Das Gebet der Kirche-The Church's Prayer-, written in 1936 when in the Carmel of Cologne, Germany, Edith Stein set forth her experience and her understanding of prayer, of liturgy and of the culmination of both in the Eucharist. This work is a spiritual testament in which the author deepens and links together, from its roots in the Jewish tradition, the meaning of prayer as an intimate and personal adhesion to Jesus Christ. Of the Eucharist as the founding experience of that communion in the Church, and of a Christian praxis in a sacrificial perspective as internal moment and necessary verification of that experience. This paper will highlight three basic contents of the text: (1.) the christian's prayer is always an act "learned" from the prayer of Christ, which is his "model" par excellence; (2.) prayer is always the Church's prayer and not only an individual act; (3.) prayer always tends to action, as an indefectible and necessary sign of what God works in the spiritual life of his faithful.

Keywords: Edith Stein - Prayer - Church - Eucharist - Action. 


\section{INTRODUCCIÓN}

Las presentes reflexiones son fruto de la lectura meditada de un escrito de Edith Stein del año 1936, Das Gebet der Kirche, publicado por primera vez al año siguiente ${ }^{1}$. El texto tiene tres partes, que abarcan diversas dimensiones de la oración cristiana: en primer lugar, la dimensión litúrgica, de por sí comunitaria y pública, con su corolario en la celebración eucarística; en segundo lugar, el "solitario diálogo" (einsame Zwiesprache) con Dios, que es, no obstante, oración "de la Iglesia"; por último, la relación que se establece mediante la oración, entre la vida interior, su exteriorización (aüsere Form) y la acción (Tat) de la libertad como consecuencia de la radicalidad de esa vida espiritual que acontece en lo más profundo del creyente.

La literatura secundaria ha insistido en el análisis complexivo de las diversas temáticas que Edith Stein articula a través de estas tres secciones. Esas temáticas tienen una vinculación muy estrecha con el contexto biográfico de la autora. Si para ella la oración tiene una fuerte dimensión de "diálogo", la presente lectura de su texto quiere ser también, a su modo, un acercamiento dialógico. En este caso, se trata del diálogo entre un lector que aborda el texto desde la teología, y la autora con su impronta personal: una mujer; una intelectual forjada en un ambiente de élite de la filosofía contemporánea, la fenomenología del círculo de E. Husserl; una filósofa alemana convertida a Cristo y a la Iglesia católica desde la tradición cultural judía. El texto refleja todo ese bagaje; un bagaje que es ante todo una experiencia personal, una historia de vida que incluye el aprendizaje, igualmente vital, de la neocristiana en los grandes centros de la renovación litúrgica alemana en la primera mitad del siglo XX: Beuron y Maria Laach².

1 En la serie Ich lebe und ihr lebet. Vom Strom des Lebens in der Kirche, ed. por la Akademischen Bonifatius-Einigung, Paderborn 1937, 69-84. En el presente trabajo utilizaré la edición alemana de Edith Stein Gesamtausgabe, Vol. 19: Geistliche Texte I, Herder, Freiburg-Basel-Wien 2014 ( $2^{a}$ Ed.), 44-58 (en adelante ESGA 19). Para citas textuales remitiré al texto alemán y también, en segundo término, a la traducción de Obras Completas, Vol. V: Escritos espirituales, Ed. de Espiritualidad-Ed. Monte Carmelo-Eds. El Carmen, Madrid 2004, 107-121 (en adelante OCES 5), que eventualmente modificaré cuando me parezca necesario.

2 Cf. MARIO GULLO, "Edith Stein: Image de l'Église en prière”, Vies Consacrées 81, no 2 (2009): 134-35. 
Pero hay algo más: Edith Stein escribe para nosotros estas reflexiones sobre la oración de la Iglesia desde la vida contemplativa ${ }^{3}$, en la cual ha venido profundizando y enriqueciendo su experiencia del cristianismo con la lectura de los grandes místicos de su tradición religiosa carmelitana y del gran padre del pensamiento cristiano escolástico medieval, Tomás de Aquino. Más de alguna vez se ha destacado el impacto que tuvieron para la joven filósofa sus primeros contactos con la comprensión tomasiana de la realidad, y en especial con su ampliación de horizontes hacia la realidad de Dios ${ }^{4}$.

Todo este contexto vital es el que se ha intentado tener presente a través de la presente lectura. Vuelvo a caracterizarla como un diálogo, aunque en este caso ha sido evidente el protagonismo de la autora. Como interlocutor casi me he limitado a acoger la riqueza de su reflexión, a ponerla de relieve y a destacar de modo especial la contundencia de su comprensión teológica del tema, que asombra aún más al constatar el hecho de que ella no llegó a recibir una formación sistemática en teología cristiana. Precisamente, una pregunta recurrente durante esta lectura ha sido cuánto ha podido aportar en este sentido su anterior pertenencia a la tradición religiosa del pueblo de la elección y la alianza. El texto ofrece, de hecho, algunas pistas al respectós.

Tres ideas-fuerza de la reflexión de Edith Stein en este escrito son las que quisiera aquí destacar: la primera es que la oración del cristiano es siempre un acto "aprendido" de la oración de Cristo, que es su "modelo" por antonomasia; la segunda, que la oración, en todas sus formas, es siempre oración de la Iglesia y no simplemente un acto individual; y la tercera, que la oración tiende siempre a una acción, pero no como

3 Sobre la inspiración carmelitana-teresiana de estas reflexiones, Cf. Jesús Castellano, “"La preghiera della Chiesa' - Una rilettura teologica", en Edith Stein: testimone di oggi, profeta per domani. Atti del Simposio Internazionale, Roma-Teresianum, 7-9 ottobre 1998 (Città del Vaticano: Libreria Editrice Vaticana, 1999), 198-202.

4 Cf. ANNELIESE MEIS, "Edith Stein y Tomás de Aquino: repercusión sobre la cuestión de la mujer", Teología y Vida LI (2010): 9-37; S. B. Sharkey, "Edith Stein and Thomas Aquinas on Being and Essence", American Catholic Philosophy Quarterly, no 82 (2008): 87-103.

5 Cf. PIERSANDRO VANZAN, "Edith Stein, filha de Israel, da Igreja, do Carmelo", Cultura e Fé 33, no 131 (s. f.): 405-15.; Castellano, "La preghiera della Chiesa” - Una rilettura teologica", 186-90. 
una simple "aplicación" en un plano secundario de utilidad, sino como elemento interno y constitutivo de la misma oración y como expresión indefectible y necesaria de la radicalidad de aquello que Dios obra en la vida espiritual de sus fieles.

\section{La ORACIÓn de la Iglesia es SIEMPre la ORACIÓn de Cristo}

Edith Stein comienza su reflexión reproduciendo, en latín en el manuscrito conservado, la doxología final de la plegaria eucarística:

"Per ipsum et cum ipso et in ipso

est tibi Deo Patri Omnipotenti

in unitate Spiritus Sancti

omnis honor et Gloria"6.

"Por Cristo, con Él y en Él, a ti, Dios Padre omnipotente, en la unidad del Espíritu Santo, todo honor y toda gloria". Con ello sitúa trinitariamente el sentido general de la oración cristiana y particularmente de su corolario en la Eucaristía ${ }^{7}$, pero poniendo su acento en el carácter cristológico y cristocéntrico de toda forma de oración: por, con y en Él nos podemos dirigir al Padre, en el Espíritu. Acota la autora: "El doble sentido del por, con y en es la clara expresión de la mediación del HombreDios". Pero no solo eso, pues, finalmente, Cristo, el Cristo Total, es la Cabeza y el Cuerpo inseparablemente unidos. Esta íntima unión de la Iglesia con Cristo permite a Edith Stein concluir con toda propiedad que "la Iglesia orante es Cristo mismo", es decir, que "la oración de la Iglesia es la oración del Cristo viviente"10.

6 La nota 2 de la edición de la ESGA, página 44, aporta este dato, apuntando además la precisión de que en la publicación misma del texto, Edith Stein puso, en lugar del latín, la siguiente traducción alemana de la doxología: "Durch Ihn, mit Ihm und in Ihm wird Dir, Gott, allmächtiger Vater, in Vereinigung mit dem Heiligen Geiste alle Ehre und Verherrlichung zuteil!".

7 Cf. DANIELE COGONI, "Das Gebet der Kirche. Fenomenlogia liturgica e spiritualita trinitaria in Edith Stein", Teresianum 67, nº 1 (2016): 133-72.

8 ESGA 19, 45 /OCES 5, 109.

9 ESGA 19, 45 /OCES 5, 108.

10 ESGA 19, 45 /OCES 5,109. 
Esta afirmación, de por sí contundente, no se queda aquí en un nivel puramente doctrinal, porque nuestra autora avanza, por así decirlo, a un nivel de significación más propiamente histórico-salvífico: la oración de la Iglesia es la oración de Cristo no solo porque ella es Su Cuerpo; lo es, más concretamente, porque, tal como lo muestran los evangelios, la Iglesia ha sabido descubrir "su modelo original (Urbild) en la oración de Cristo durante su vida terrena" ${ }^{11}$. Este "modelo" brilla ante todo por su inserción en la historia y en la tradición creyente de un pueblo, lo cual justifica que una primera profundización acerca de la oración sea su dimensión litúrgica y, en definitiva, eucarística ${ }^{12}$.

\section{LitURGIA Y EUCARISTÍA: LA IGLESIA COMO “LUGAR” DE TODA ORACIÓN}

La primera lección que la Iglesia, según los testimonios evangélicos, aprende de Cristo es su actitud orante como "un judío creyente y fiel a la Ley"13. Para Edith Stein es especialmente significativo imaginar o contemplar este aspecto en la vida del Nazareno: aprendiendo a orar con sus padres como todo niño del pueblo elegido; peregrinando a Jerusalén tantas veces durante su vida oculta y, más tarde, con sus discípulos; cantando en esas ocasiones los himnos y salmos que ensalzaban la alegría de la alabanza a Dios; bendiciendo el pan y dando gracias por él en cada ocasión de comensalidad.

Esta imagen es la que nuestra autora tiene como trasfondo al hacer presentes los gestos y las palabras de Jesús en su última cena con sus discípulos. Edith Stein no duda en ver en esta escena el reflejo de una cena pascual que los discípulos solo más tarde, a la luz de la propia Pascua de Jesús, sabrían comprender en toda su profundidad y significación. Los así llamados "relatos de institución" reflejan esa mezcla de resabios israelitas y resignificación cristiana de lo que Jesús dijo e hizo en esa cena impregnada por la inminencia del conflicto final de su existencia entregada, con total radicalidad, al anuncio del reinado de Su Padre. Edith Stein aparece aquí

\footnotetext{
11 ESGA 19, 45 /OCES 5, 109.

12 Cf. Marie-Jean De la Rédemption, "Un témoin de l'Église orante: Édith Stein", Carmel 82, n 4 (1996): 45-62.

13 ESGA 19, 45 /OCES 5, 109.
} 
con especial intensidad como la judía convertida al cristianismo: "La bendición y el repartir (Verteilung) el pan y el vino eran parte del rito de la cena pascual. Pero ambas reciben aquí un sentido completamente nuevo. Con ellas comienza la vida de la Iglesia" ${ }^{14}$. Al respecto, resulta sugerente la tesis de C. Betschart en el sentido de que Edith Stein considera a la última Cena, en cuanto institución de la Eucaristía, como el origen propio de la Iglesia, más que a Pentecostés y más que al mismo sacrificio de Jesús en la cruz, para acentuar su convicción de que no es posible comprender plenamente a la Iglesia sin considerar la antigua alianza. ${ }^{15}$

A partir de esta mirada, comienza una sección especialmente vibrante del texto. En primer lugar, Edith Stein sitúa su lectura de la última cena en la perspectiva hermenéutica de otro gran converso, Pablo de Tarso, tal como este se expresa en Rom 11,13-24 $4^{16}$ con la imagen de otro "injerto", el de las ramas de olivo silvestre en el olivo cultivado, como símil del surgir histórico de la Iglesia desde las raíces de Israel, el pueblo de la gratuita elección divina (Dt 9,6; 7,8) y de la alianza con Él (Ex 19,3-8; 24,3-8). Para nuestra autora, la última cena de Jesús con sus discípulos, que ella entiende derechamente como pascual, es el momento en que tiene lugar "el injerto de los sarmientos en la vid (Weinstock), que hace posible la efusión del Espíritu. Las antiguas oraciones de bendición se han convertido en boca de Cristo en palabra creadora de vida" ${ }^{17}$. Lo que sigue es una sorprendente relectura de la última cena testimoniada en el Nuevo Testamento ${ }^{18}$. Sorprendente, como decía anteriormente, por la contundencia teológica de su análisis. Someramente, destaco algunos tópicos de esta relectura tan personal.

En primer lugar, una vívida teología de la creación: "los frutos de la tierra se han convertido en carne (Fleisch) y sangre, llenos de su vida. La creación visible en la que entró ya por su encarnación (Menscbwerdung), está

\footnotetext{
14 ESGA 19, 46 /OCES 5, 109.

15 Cf. Christof Betschart, "La responsabilité de la prière dans l'ecclésiologie d'Edith Stein", Nowvelle Revue Théologique 138, no 4 (2016): 592-93; Cogoni, "Das Gebet der Kirche. Fenomenlogia liturgica e spiritualita trinitaria in Edith Stein”, 141-53.

16 Que, empero, nuestra autora no cita aquí explícitamente.

17 ESGA 19, 46/ OCES 5, 109s.

18 Cf. Cogoni, "Das Gebet der Kirche. Fenomenlogia liturgica e spiritualita trinitaria in Edith Stein”, 153-57.
} 
ahora unida a él de un modo nuevo, misterioso"19. La creación, enaltecida por la Encarnación (de ahí que utilice aquí la expresión carne), ahora entra en una dimensión nueva y más profunda.

Habla luego de Stoffe, término que en las Obras Completas se traduce por "sustancias". Si bien es cierto Edith Stein no recurre aquí al término técnico filosófico Substanz, es bastante claro, por el contexto, que está trayendo a colación un concepto de amplia relevancia en la teología eucarística católica. De hecho, en seguida agrega que aquellas sustancias "son transformadas (sind umgewandelt) radicalmente y por su recepción creyente se transforman también los hombres, incorporados a una unidad de vida con Cristo y llenos de su vida divina" ${ }^{20}$. El tema de la vida, y de la vida divina, aparece aquí de modo especial a propósito de la sangre derramada que el fruto de la vid viene a significar, con los gestos y las palabras de Jesús, como acontecimiento salvífico mediante Su vida entregada. Tal es, por lo demás, el núcleo de la resignificación cristiana del sacrificio pascual que se realiza en los relatos evangélicos de institución, y que se expresa de modo especialmente claro mediante el cáliz que el Maestro "da" y distribuye a sus discípulos en este postrero encuentro comensal antes de su pasión ${ }^{21}$.

Estas palabras de Jesús en su última cena contienen, pues, para Edith Stein, "la fuerza de la Palabra creadora", y esta fuerza está vinculada al sacrificio. Aparece así, por primera vez en el texto, de manera patente, la dimensión práxica del acto redentor como acto de ofrenda, y de la comunión de la Iglesia y de cada creyente con ese acto: si "la Palabra se hizo carne para ofrecer la vida que recibió" 22 , eso conmueve y mueve a los cristianos. De este modo entiende Edith Stein el que en esta cena "la comida pascual de la Antigua Alianza se ha convertido en la comida pascual de la Nueva Alianza"23, es decir, en un nuevo y definitivo vínculo con el Dios de la vida. El sacrificio, que está significado en los gestos y palabras de Jesús, expresa el sentido total de su existencia, y ello marca el camino a seguir

\footnotetext{
19 ESGA 19, 46 /OCES 5, 110.

20 ESGA 19, 46 /OCES 5, 110.

21 Mc 14,23 y Mt 26,27; de modo menos explícito, pero igualmente claro, en Lc 22,20 y 1Co 11,25.

22 ESGA 19, 46 /OCES 5, 110.

23 ESGA 19, 46 /OCES 5, 110.
} 
también por sus discípulos. La última cena, entendida como institución de la Eucaristía, solo podrá ser revivida y comprendida en toda su profundidad por los discípulos mediante su inserción en el contexto de una vida eucarística ${ }^{24}$.

De nuevo nos podemos preguntar, con nuestra autora: ¿dónde acontece, realmente, el sacrificio de la Nueva Alianza? Responde Edith Stein: "en el sacrificio de la cruz del Gólgota y en aquellas comidas gozosas del tiempo entre Pascua y Ascensión, en las que los discípulos reconocían al Señor al partir el pan, y en el sacrificio de la misa"25. Es decir, es el sacrificio uno y único que empero se ofrece también en las diversas dimensiones de la presencia de Cristo. Una especial novedad en esta comprensión del origen del sacramento de la Eucaristía en Cristo, es que nuestra autora no se limita a la escena de la última cena, que sintetiza y compendia toda una historia de comensalidad durante la existencia terrena de Jesús junto a sus discípulos, sino que también sabe reconocer su raíz en las comidas de los discípulos con el Resucitado, esas situaciones en que el Señor se deja reconocer "en la fracción del pan" (Lc 24,30s.35) ${ }^{26}$.

A propósito de la acción de gracias que Jesús hace sobre el cáliz, nuestra autora de nuevo remite al gesto creyente de todo buen judío en el momento de la comensalidad, pero además lo vincula con el antecedente, que está en el conjunto de la vida de Jesús, de elevar su mirada y su gratitud al Padre cada vez que hacía un signo de la irrupción de Su reinado, "por la fuerza divina de que es portador y porque va a mostrar ante los ojos de los hombres la omnipotencia del Creador" ${ }^{27}$. Ahora esa acción de gracias cobra una nueva e inconmensurable dimensión, esto es, la de una "acción de gracias por la creación, la redención y la plenitud. Cristo se ofrece a sí mismo en nombre de toda la creación, cuyo prototipo es él y a la que ha descendido a fin de renovarla desde dentro y llevarla a la plenitud" ${ }^{28}$.

\footnotetext{
24 Cf. Gullo, "Edith Stein: Image de l'Église en prière", 141-43.

25 ESGA 19, 46 /OCES 5, 110.

26 Sobre este importante aspecto de la comprensión teológica actual del origen de la Eucaristía en el Jesús histórico, cf. el clásico estudio de Ferdinand Hahn, "Alttestamentlichen Motive in der urchristlichen Abendmahlsüberlieferung", Evangelische Theologie, $\mathrm{n}^{\circ} 27$ (1967): 337-74.

27 ESGA 19, 46 /OCES 5, 110.

28 ESGA 19, 47 /OCES 5, 110.
} 
Este aspecto cósmico de la acción de gracias, de la eu-caristía, llama a Edith Stein a una última reflexión sobre el modo como ello se expresó, en la tradición de Israel, en torno a una teología del templo como "símbolo de toda la creación que se reúne en adoración y servicio en torno al Señor"29. En continuidad con esa visión, pero también en completa novedad, Edith Stein ve cómo "Cristo ha construido un templo de piedras vivas", que es "la comunión de los santos"30. Este es el verdadero templo en cuyo altar Cristo realiza el sacrificio como "eterno y sumo sacerdote", a la vez que como "víctima perpetua". La cita de Hebreos es implícita, pero evidente.

En esta gran liturgia no solo toma parte toda la creación, sino que además las oraciones que en ella se hacen alcanzan, "envuelven y santifican todo el trabajo del día, de modo que de la oración y del trabajo resulta un solo 'opus Dei', una sola 'liturgia"' ${ }^{31}$. En esta confluencia de toda la creación, incluidos los ángeles y los santos, y del trabajo del hombre, es convocado, para Edith Stein, el gran "nosotros", que ella entiendeanticipando así una eclesiología todavía en ciernes- constituido "no solo [por] los religiosos cuyo oficio es la solemne alabanza divina, sino [por] todo el pueblo cristiano... [que] entonces muestra que es consciente de su vocación a la alabanza divina" ${ }^{32}$. Solo así podrá darse lo que ella llama "la unidad litúrgica de la Iglesia del cielo y de la Iglesia de la tierra..."

La expiación, dado que el sacrificio es también sacrificio de expiación, se ordena, en todo caso, a que podamos ser recibidos en "la comunión (Gemeinschaft) de los espíritus bienaventurados", mediante la única lógica necesaria, la de la plegaria del Padrenuestro ${ }^{34}$. Solo de ese modo el pan eucarístico será para nosotros, verdaderamente, "el pan de vida que necesitamos cada día para ir creciendo y adentrando en la vida eterna" ${ }^{35}$. El resultado de la participación en el sacrificio y en la comunión es que se convierte el alma "en piedra viva de la ciudad de Dios, y a cada alma en

\footnotetext{
29 ESGA 19, 47 /OCES 5, 110.

30 ESGA 19, 47 /OCES 5, 111.

31 ESGA 19, 48 /OCES 5, 111.

32 ESGA 19, 48 /OCES 5, 112.

33 ESGA 19, 48 /OCES 5, 112.

34 ESGA 19, 49 /OCES 5, 112.

35 ESGA 19, 49 /OCES 5, 113.
} 
un templo de Dios" ${ }^{36}$. En esta dimensión de la oración como espacio de intimidad y hondura, Cristo también es el modelo.

\section{DE LA VIDA INTERIOR A LA ACCIÓN}

Edith Stein observa que los relatos evangélicos no solo nos muestran a Jesús como creyente fiel y activo participante del culto público, sino también como un hombre de oración solitaria, extensa y profunda; "oración solitaria en el silencio de la noche, en la libre cumbre de la montańa, en el desierto alejado de los hombres" ${ }^{37}$. La pluma de nuestra autora se desliza con soltura trayendo a su reflexión escenas evangélicas que vienen a ilustrar esta idea.

Destaca el hecho de que esta actitud orante se observa en Jesús no solo en los momentos en los que se aparta de la muchedumbre y de la presencia de sus discípulos, sino también estando entre la gente. Y de esas situaciones resalta una, en la que Edith Stein entiende que Jesús "nos permitió mirar larga y profundamente el secreto de ese íntimo diálogo" con su Padre ${ }^{38}$. Se refiere a la así llamada oración sacerdotal de Jesús, en Jn 17. La relaciona con el Día de la Reconciliación (Yom Kippur) descrito en el Primer Testamento (Lev 16) como "prefiguración del viernes santo", y destaca cómo en esta oración Jesús "desvela el misterio del sumo sacerdocio" 39 . Esto sus discípulos pueden presenciarlo y experimentar así otro aprendizaje. Ellos, observa la autora, "deben comprender de qué se trata (worum es geht), y deben aprender a hablar en su corazón con el Padre" ${ }^{40}$.

Y de lo que se trata es aquello que la oración sacerdotal de Jesús, en definitiva, manifiesta: "el misterio de la vida interior: la inmanencia recíproca (das Ineinander) de las Personas divinas y la inhabitación (Innewobnen) de Dios en el alma"41. Es en ese espacio íntimo y profundo donde se ha fraguado, en el silencio, la obra de la redención. Edith Stein contempla

ESGA 19, 49 /OCES 5, 113.

ESGA 19, 50 /OCES 5, 113.

ESGA 19, 50 /OCES 5, 114.

ESGA 19, 51 /OCES 5, 115.

ESGA 19, 51 /OCES 5, 115.

41 ESGA 19, 51 /OCES 5, 115. El destacado en cursiva es de Edith Stein.
} 
en esta clave todo el mensaje evangélico, y ya no solo la oración de Jesús. Contempla a María en la Anunciación (Lc 1,26-38); a Saulo cuando espera del Señor, en oración, total claridad para saber cómo servirlo (Hch 9); a Pedro preparando en el silencio de la oración su anuncio del Mensaje (Hch 10); a María, nuevamente, que guarda en su corazón los acontecimientos, los de su maternidad de Jesús, en que Dios se manifiesta (Lc 2,19.51)...

De un modo algo abrupto, en este momento de su reflexión Edith Stein comienza a destacar la figura de otras cristianas, que, como María, "se olvidaron completamente de sí mismas para sumergirse en la vida y en la pasión de Cristo..." "42: Santa Brígida (copatrona de Europa, junto con la misma Edith Stein que aquí la homenajea), Catalina de Siena (la tercera copatrona); y, por supuesto, santa Teresa de Ávila. De su ejemplo, Edith Stein toma el fundamento que necesitaba para destacar, por último, la necesaria repercusión de la oración en la vida del creyente: el terreno de la acción.

Esta dimensión nuestra autora la destaca como manifestación inequívoca de que lo que el Espíritu obra en lo profundo de la vida interior puede llevar a consecuencias imprevisiblemente significativas en el ámbito existencial. Se trata de una obra, la obra del Espíritu, que puede desencadenar una corriente, la "corriente mística" que impregna la vida misma de la Iglesia. Y -reflexiona Edith Stein- "cuando rompe con las formas tradicionales, lo hace porque vive en ella el espíritu que sopla donde quiere: el Espíritu que ha creado todas las formas tradicionales y que tiene que crear continuamente formas nuevas. Sin él no habría ni liturgia ni Iglesia" $"$.

\footnotetext{
42 ESGA 19, 52-53 /OCES 5, 116.

43 ESGA 19, 55 /OCES 5, 118.
} 


\section{Epílogo: La ORACión, la EuCARISTía Y UNA EXISTENCIA EUCARÍstica}

Otros ejemplos de personas santas pueden venir aquí a reforzar esta convicción de Edith Stein: que la plenitud humana no tendrá mejor realización que la entrega confiada de la voluntad humana a la voluntad divina, conocida en la profundidad de la oración. Se trata aquí de una entrega ilimitada, que solo puede ser expresión del amor, amor que busca "la unión completa y duradera". Y ello "es la suprema elevación del corazón que nos es posible alcanzar, el supremo grado de oración. Las almas que lo han alcanzado son verdaderamente el corazón de la Iglesia"’ł. Para Edith Stein, "esta victoria del poder divino sobre la libertad humana, a la que Él a pesar de todo deja actuar, es uno de los más admirables y adorables aspectos del plan divino sobre el mundo" 45 .

Al final de la reflexión, Edith Stein vuelve la mirada a Jesús el Cristo, porque Él es "el camino hacia la vida interior" ${ }^{46}$. El último párrafo del texto es una verdadera catequesis sobre los sacramentos de iniciación, con el valor adicional de reflejar una meditación profunda sobre ellos por parte de una convertida excepcional. Del Bautismo destaca su carácter purificador y penitencial, mencionándolo junto con el sacramento de la reconciliación; de la Confirmación destaca su "marca" y su fortalecimiento del "soldado de Cristo", es decir, su ordenamiento al testimonio creyente en el mundo; finalmente, de la Eucaristía destaca la recepción del pan de vida como participación en el sacrificio y en la comunión, pero con un clarísimo acento en la finalidad de asegurar la unidad de cada fiel y de la Iglesia como un todo con Cristo. Citando a Agustín, reflexiona: "Nada debe temer tanto el cristiano [...] como la separación del cuerpo de Cristo. Porque cuando se separa del cuerpo de Cristo, ya no es miembro, y si no es su miembro ya no lo vivifica el Espíritu" ${ }^{47}$. Y completa esta reflexión con palabras de san Juan Crisóstomo:

\footnotetext{
44 ESGA 19, 55-56 /OCES 5, 119.

45 ESGA 19, 56 /OCES 5, 120.

46 ESGA 19, 57 /OCES 5, 121.

47 S. Agustín de Hipona, Tractatus 27 in Joaannem [PL., 35, 1618]. Breviario romano, tercer día en la octava de Corpus, lecturas 8 y 9, citado en ESGA 19, 57 /OCES 5, 121.
} 
"Nos convertimos en miembros del cuerpo de Cristo 'no solo por el amor..., sino realmente por la incorporación a su carne; esto se realiza mediante la comida que nos regaló para mostrar su amor a nosotros. Para esto vino a nosotros y conformó su cuerpo al nuestro, para que seamos uno, como el cuerpo se une con la cabeza..." ${ }^{48}$

En esta perspectiva se hace aún más manifiesto el núcleo de lo que la autora ha querido transmitir a través de todo este texto: su convicción de que la oración, y sobre todo la oración cúspide que es la eucaristía, es un camino de unión con Dios en Cristo que, para cada cristiano y para la Iglesia toda, no puede sino concretarse, hacerse carne, en el ámbito de la acción; esto es: en el concreto despliegue de la existencia humana como praxis de una vida entregada, en imitación y seguimiento de Jesucristo que entrega su cuerpo y su sangre -su vida, su persona- como "pan de vida y bebida de salvación". El discipulado del Resucitado, que no es otro que el Crucificado, constituye el camino por antonomasia para cada cristiano y para toda la Iglesia. A ese Cristo es que la Iglesia está llamada a unirse como se une el cuerpo a su Cabeza (Col 1,18; Ef 4,11-16), la esposa al Esposo (Ef 5,25-32). ${ }^{49}$

Edith Stein, en el momento decisivo de la muerte, ya como Teresia Benedicta a Cruce, debió experimentar en su máxima radicalidad la convicción fundante de los cristianos acerca del sentido de la existencia humana y que está contenida, precisamente, en la doxología final de la plegaria eucarística con la que nuestra autora daba inicio a este texto sobre la oración de la Iglesia y sus consecuencias:

"Per ipsum et cum ipso et in ipso

est tibi Deo Patri Omnipotenti

in unitate Spiritus Sancti

omnis honor et Gloria”.

S. Juan Crisóstomo, Homilía 61 ad populum Antioch. Breviario romano, tercer día en la octava de Corpus, lectura 4, citado en ESGA 19, 57-58 /OCES 5, 121.

49 Para profundizar en este aspecto eclesiológico del pensamiento de Edith Stein, cf. Denis Chardonnens, "Edith Stein et le mystère de l'Église", Teresianum LI, no 1 (2000): 57-83. 